\title{
Association study of the G-protein signaling 4 (RGS4) and proline dehydrogenase (PRODH) genes with schizophrenia: a meta-analysis
}

\author{
Dawei $\mathrm{Li}^{1,2}$ and $\mathrm{Lin} \mathrm{He}^{\star, 2,3}$
}

\author{
${ }^{1}$ Bio-X Life Science Research Center, Shanghai Jiao Tong University, Shanghai, China; ${ }^{2}$ Institute for Nutritional \\ Sciences, Shanghai Institutes of Biological Sciences, Chinese Academy of Sciences, Shanghai, China; \\ ${ }^{3}$ NHGG Bio-X Center, Shanghai Jiaotong University, Shanghai, China
}

Schizophrenia is a devastating psychiatric disease that affects up to $1 \%$ of the population worldwide. Recent studies suggested that schizophrenia might result from the hypofunction of glutamatergic neurotransmission. Systematic positional, expression and functional studies have implicated the regulator of G-protein signaling 4 (RGS4) and proline dehydrogenase (PRODH) genes as promising and novel candidates for explaining schizophrenia. However, the findings of association studies tend to vary depending on the different populations on which they have been conducted. To reconcile this conflict of evidence, we combined all available population-based and family-based studies up to July 2005 involving eight polymorphisms. However, this meta-analysis did not find statistically significant evidence for association between the two glutamate-related genes and schizophrenia on the basis of either allelic or genotypic analysis. This may be the first systematic meta-analysis study based on RGS4 and PRODH. European Journal of Human Genetics (2006) 14, 1130-1135. doi:10.1038/sj.ejhg.5201680; published online 21 June 2006

Keywords: meta-analysis; schizophrenia; association; G-protein signaling 4 (RGS4); proline dehydrogenase (PRODH)

\begin{abstract}
Introduction
Schizophrenia is a devastating psychiatric disease that affects up to $1 \%$ of the population worldwide. ${ }^{1,2}$ Studies suggest that schizophrenia might result from the hypofunction of glutamatergic neurotransmission. ${ }^{3,4}$ Systematic linkage scans and review studies have identified several promising and novel 'positional candidates', including the regulator of G-protein signaling 4 (RGS4) and proline dehydrogenase $(P R O D H)$ genes. ${ }^{5-8}$ The RGS4 gene maps to $1 \mathrm{q} 21-\mathrm{q} 22$, a candidate region that is close to a linkage peak. ${ }^{9}$ RGS4 is a negative regulator of G-protein-coupled
\end{abstract}

*Correspondence: Professor L He, Bio-X Center, Shanghai Jiaotong University, Hao Ran Building, 1954 HuaShan Road, Shanghai 200030, People's Republic of China; or Institute for Nutritional Sciences, SIBS, Chinese Academy of Sciences, 294 Taiyuan Road, Shanghai 200031, China. Tel \& fax: +8621 62822491; E-mail: helin@sjtu.edu.cn Received 19 September 2005; revised 4 April 2006; accepted 16 May 2006; published online 21 June 2006 receptors, including the metabotropic glutamate receptor, ${ }^{5,10}$ and the $R G S$ family are a group of GTPase-activating proteins that are abundant in brain regions implicated in schizophrenia, such as the neocortex, the caudate and the putamen. The expression of RGS4 has been shown to be downregulated in the postmortem brains of schizophrenic patients $^{11}$ and to interact with ErbB3, ${ }^{12}$ which is also differentially expressed in the brains of schizophrenic patients. ${ }^{13}$ The ErbB proteins family acts as receptors for neuregulin 1, which itself has been confirmed as a susceptibility gene to schizophrenia. ${ }^{14}$ The RGS4 gene is, therefore, a positional, expression and functional candidate for schizophrenia. Chowdari et al ${ }^{15}$ first reported the association with schizophrenia. The four single-nucleotide polymorphisms (SNPs) (SNPs 1, 4, 7 and 18) in the associated haplotype are non-coding SNPs, but SNPs 1, 4 and 7 are located in $5^{\prime}$ region of the gene, which may play a role in transcription regulation. 
The PRODH gene, consisting of 14 exons, is also likely to be a promising candidate in terms of its physical location, function and genetic linkage evidence on chromosome 22q11.2. Deletion of the $22 q 11$ region associated with Velo cardio-facial syndrome constitutes one of the highest risk factors for schizophrenia and confers a 20-30-fold increase in risk of the disease. ${ }^{6,16-19} P R O D H$ is widely expressed in the brain and other tissues, ${ }^{20}$ and its product is localized within the mitochondria where it catalyses the conversion of proline to D-1-pyrroline-5-carboxylate which can then be converted to glutamate or $g$-aminobutyric acid, both of which are candidate neurotransmitters for schizophrenia. ${ }^{21} \mathrm{Liu}$ et al $^{22}$ have recently reported association between $P R O D H$ and schizophrenia using three sets of independent samples.

However, the evidence on association varied for each gene depending on the population used. To clarify this inconsistency and to establish whether there was an association between the common polymorphisms of each gene and schizophrenia, the current meta-analysis has combined data from all relevant published populationbased and family-based association studies.

\section{Methods}

\section{Literature search}

The literature included in the analysis was selected using PubMed and focused on the keywords 'schizophrenia' 'regulator of G-protein signaling 4' 'proline dehydrogenase' and abbreviation of the genes 'RGS4' 'PRODH'. All references cited in these studies and published reviews were reviewed in order to identify additional works not indexed by MEDLINE. The analyzed data cover those from all English language publications up to July 2005.

\section{Inclusion criteria}

Eligible studies had to meet all of the following criteria namely that: (1) they were published in a peer-reviewed journal and were independent studies using original data, (2) they provided sufficient data to calculate the odds ratio (OR) with confidence interval (CI) and $P$-value, (3) they investigated one or more of the eight polymorphisms using either population-based or family-based approaches, (4) they described the genotyping primers, machines and protocols or provided reference to them, (5) they diagnosed schizophrenia patients according to the ICD, DSM or Chinese classification of mental disorders systems and (6) they used healthy individuals as controls. Authors were contacted in cases where there were queries regarding their studies.

\section{Assessments of quality: extended quality score}

For association studies with inconsistent results on the same polymorphisms, the methodological quality needs to be assessed using appropriate criteria to limit the risk of bias in the meta-analysis. The technique known as 'Extended-Quality Score' (Ver1.1) was used to assess the quality of association studies under which each paper was scored as being of 'high', 'median' or 'poor' quality.

\section{Statistical analyses}

Any study containing data from different ethnic populations was considered effectively as a series of individual studies. Data from the case-control and haplotype-based haplotype relative risk (HHRR) studies were summarized in two-by-two tables and transmission disequilibrium test (TDT) studies were summarized in two-by-one tables. From each table, a log-OR and its sampling variance were calculated. ${ }^{23}$ Cochran's $\chi^{2}$-based $Q$ statistic test was performed in order to assess possible heterogeneity between the individual studies. Heterogeneity $Q$ tests were also performed for differences in OR between design types (case-control $v s$ family-based). A test for funnel plot asymmetry, described by Egger et al, ${ }^{24}$ was used to assess evidence for publication bias. ORs were pooled using the method of DerSimonian and Laird, and 95\% CIs were constructed using Woolf's method. The significance of the overall OR was determined by the $Z$-test. For the sensitivity analysis, each study was removed in turn from the total, and the remaining studies were reanalyzed. This procedure was used to ensure that no individual study was entirely responsible for a finding. The type I error rate was set at 0.05. $P$-values were two-tailed. An R-project program was used to depict the degree of differences and trend of association of risk allele frequency from controls to patients. If the vector arrow had the same direction this indicated the same kind of association, and vice versa.

Haplotype construction, counting and linkage disequilibrium (LD) block defining were performed using 30 CEPH trios (Utah residents) on Haploview software (www.hapmap.org). The multiallelic $D^{\prime}$ was computed by performing a series of pairwise $D^{\prime}$ calculations using each haplotype in turn as an allele, with all other haplotypes at the locus serving as the other allele. This was then repeated for each haplotype at each locus and averaged by haplotype frequency. Maximum likelihood haplotype blocks were calculated using an Expectation and Maximization algorithm.

\section{Result}

The combined search yielded at least 67 references. After discarding overlapping references and those that clearly did not meet the criteria, 18 studies were retained. These studies were then filtered to ensure conformity with the inclusion criteria. For the RGS4 gene, one study ${ }^{25}$ was discarded for insufficient and equivocal data. For the $P R O D H$ gene, two ${ }^{26,27}$ for insufficient and equivocal data (although we tried to contact the authors to query the data) and two ${ }^{28,29}$ for non-association design studies. 
Table 1 Allelic results of all studies for each polymorphism

\begin{tabular}{|c|c|c|c|c|}
\hline Genes/markers & OR $(95 \% \mathrm{Cl})$ & $P(Z)$ & $P(Q)$ & $P(Q)^{a}$ \\
\hline $\begin{array}{l}P R O D H^{\mathrm{e}} \\
1945 \mathrm{C} / \mathrm{T}^{\mathrm{b}}(5) \\
2026 \mathrm{C} / \mathrm{T}(3) \\
\mathrm{A} 472 \mathrm{~T}(3)\end{array}$ & $\begin{array}{l}0.96(0.84,1.09) \\
0.83(0.65,1.05) \\
0.47(0.14,1.58)\end{array}$ & $\begin{array}{l}0.51 \\
0.1227 \\
0.2213\end{array}$ & $\begin{array}{l}0.4427 \\
0.9095 \\
0.0426\end{array}$ & $\begin{array}{l}\text { NS } \\
\text { NS } \\
\text { NS }\end{array}$ \\
\hline
\end{tabular}

$P(Z): Z$-test used to determine the significance of the overall $\mathrm{OR}$.

$P(Q)$ :Cochran's $\chi^{2}$-based $Q$ statistic test used to assess the heterogeneity.

$P(T)$ : $t$-test used to evaluate the significance of publication bias. No $P(T)<0.05$ (not shown).

a Heterogeneity between design types (case-control vs family-based), NS = not significant.

${ }^{\mathrm{b}}$ The first allele was the risk allele.

'The number of studies included are indicated in parentheses.

${ }^{\mathrm{d}}$ Results of the combined studies when the data of the initial association study ${ }^{15}$ were excluded.

${ }^{\mathrm{e}}$ For R431H (2), OR $(95 \% \mathrm{Cl})=1.11(0.87,1.42) ; P(Z)=0.391 ; P(Q)=0.7245$.

Finally, 13 studies, composed of three case-control studies $^{30-32}$ and three TDT studies ${ }^{15,32}$ for RGS4 (1176 cases, 1517 controls and 211 trios/sib-pairs) and seven studies (including one HHRR and one TDT) ${ }^{18,22,33-35}$ for PRODH (1428 cases, 1318 controls and 332 trios), met our criteria for inclusion. The 13 studies included 2604 cases, 2835 controls and 543 parent-offspring trios/sib-pairs and all fell within the medium/high categories of the Extended Quality Score technique.

Overall, neither the allelic (Table 1) nor the genotypic (Table 2) data in the meta-analysis showed any statistically significant association for either the RGS4 or PRODH genes, nor was any publication bias found with regard to any of the eight polymorphisms (no $\mathrm{p}(\mathrm{T})<0.05$ ). However, for the allelic analysis, evidence of heterogeneity was found in RGS4 SNP1 $(P=0.009)$, SNP4 $(P=0.0004)$ and SNP18 $(P=0.009)$, and weak heterogeneity was found in $P R O D H$ A472T $(P=0.04)$ (Table 1$)$. There was no evidence of heterogeneity between design types (case-control vs TDT) $(P>0.05)$ (Table 1). Furthermore, when we analyzed the case-control and TDT studies separately or clumped the patients by age (for $1945 \mathrm{C} / \mathrm{T}$ and $2026 \mathrm{C} / \mathrm{T}$ ), no significance was found (Table 2). The forest plots are shown in Figures 1 and 2 for the allelic analyses of RGS4 and $P R O D H$, respectively.

\section{Retrospective analysis}

The asymptote lines of the retrospective analysis based on the publication year showed that cumulative synthesis of the SNPs investigated currently tended not to be stable as revealed by asymptotic slopes (Figure 3), indicating that more replications were needed.
Table 2 Results of the studies sub-grouped by age and results of genotypic analysis

\begin{tabular}{|c|c|c|c|}
\hline Markers/types & OR $(95 \% \mathrm{Cl})$ & $P(Z)$ & $P(Q)$ \\
\hline \multicolumn{4}{|l|}{ RGS4 } \\
\hline \multicolumn{4}{|l|}{ SNP1 (A/G) ${ }^{\mathrm{a}}(6)$} \\
\hline$(11+12) / 22$ & $1.06(0.88,1.27)$ & 0.5482 & 0.6068 \\
\hline $11 /(12+22)$ & $1(0.79,1.25)$ & 0.9759 & 0.6852 \\
\hline \multicolumn{4}{|l|}{ SNP4 (T/G) (6) } \\
\hline$(11+12) / 22$ & $1.18(0.95,1.45)$ & 0.1267 & 0.2829 \\
\hline $\begin{array}{l}11 /(12+22) \\
\text { SNP7 (G/A) (4) }\end{array}$ & $1.15(0.95,1.4)$ & 0.1531 & 0.1395 \\
\hline$(11+12) / 22$ & $0.96(0.76,1.22)$ & 0.7591 & 0.6332 \\
\hline $11 /(12+22)$ & $0.94(0.78,1.12)$ & 0.4752 & 0.4127 \\
\hline \multicolumn{4}{|l|}{ PRODH } \\
\hline \multicolumn{4}{|l|}{ Age $<18^{b}$} \\
\hline 1945C/Т (4) & $0.82(0.58,1.15)$ & 0.2475 & 0.0803 \\
\hline
\end{tabular}

The results of genotypic analyses of SNP18 of RGS4 and polymorphisms of $P R O D H$ are not shown because of insufficient data.

${ }^{a}$ The first allele was the risk allele, $1=$ the first allele.

${ }^{b}$ For $2026 \mathrm{C} / \mathrm{T}(2)$, OR $(95 \% \mathrm{Cl})=0.87(0.41,1.86) ; P(Z)=0.7196$; $P(Q)=0.7627$.

The. funnel plots and trend of allele frequency by $R$ project are shown as supplements. Lack of space precluded the inclusion of the results of individual studies (available on request).

\section{Discussion}

Association between the two genes and schizophrenia was supported by studies based on individual locus or haplotype analysis, whereas other studies reported negative findings. For RGS4, different susceptible alleles were 
RGS4 In(OR)(95\% Cl)

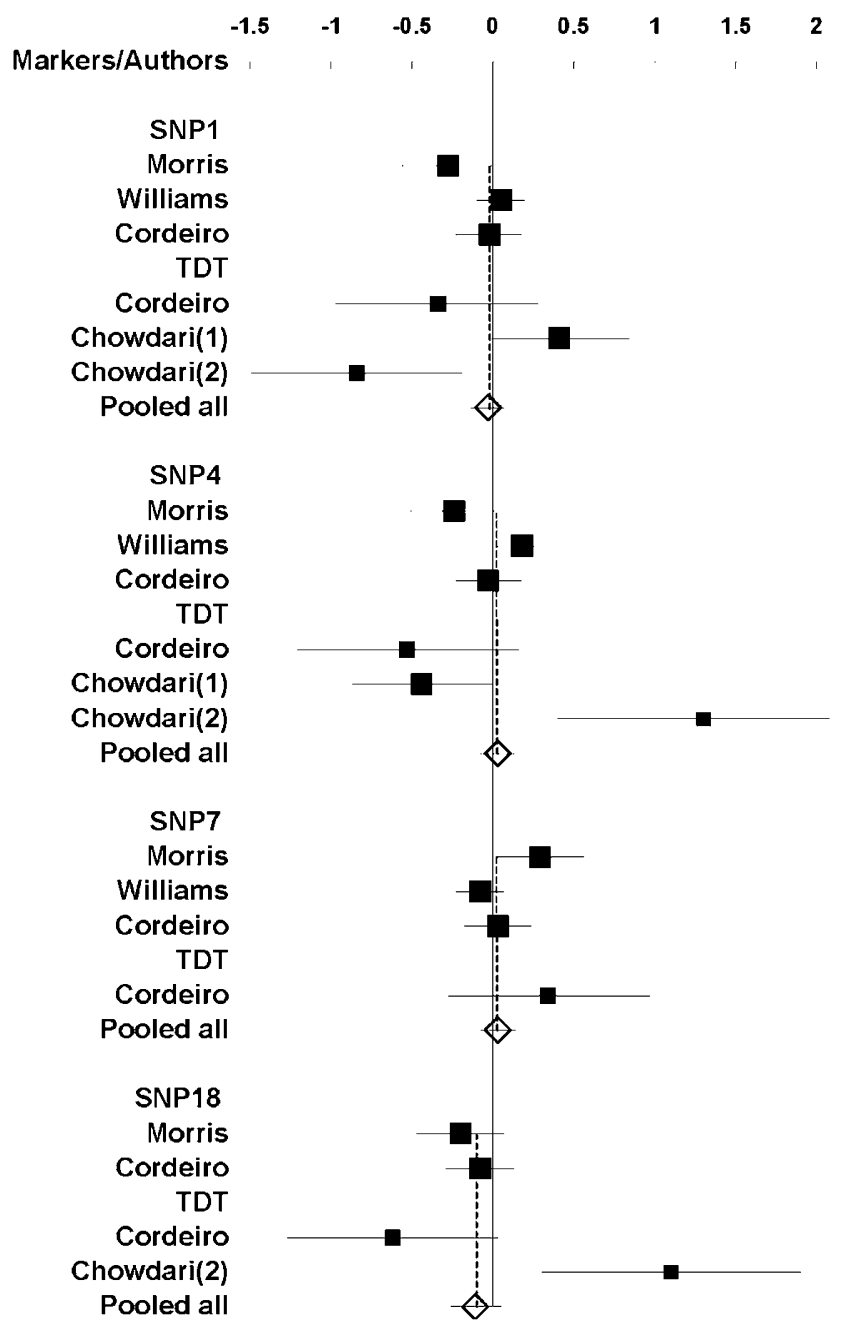

Figure 1 Forest plots of $\ln (\mathrm{OR})$ with $95 \% \mathrm{Cl}$ for each polymorphism of the allelic analysis for the RGS4 gene. Black squares indicate the $\ln (\mathrm{OR})$, with the size of the square inversely proportional to its variance, and horizontal lines represent the $95 \% \mathrm{Cls}$. The pooled results are indicated by the unshaded black diamond.

detected, and the risk allele frequencies were in different directions in different populations (four studies ${ }^{15,30,32}$ reported the $\mathrm{G}$ allele as the risk allele but two studies ${ }^{15,31}$ found it had the protective effect). Several possible reasons may exist for the observed discrepancies. Firstly, they may be attributable to sampling bias, including population stratification bias owing to the variations of ethnicities or diagnostic methods and the differences in allele frequencies. Secondly, in the initial report on RGS4, the Pittsburgh sample revealed overtransmission of the $G$ allele in all four SNPs, ${ }^{15}$ although the Pittsburgh sample size was small, which may overestimate the true effect of the gene (the 'winner's curse' problem ${ }^{36}$ ). In addition, the combined studies of RGS4 showed that the heterogeneity was weak

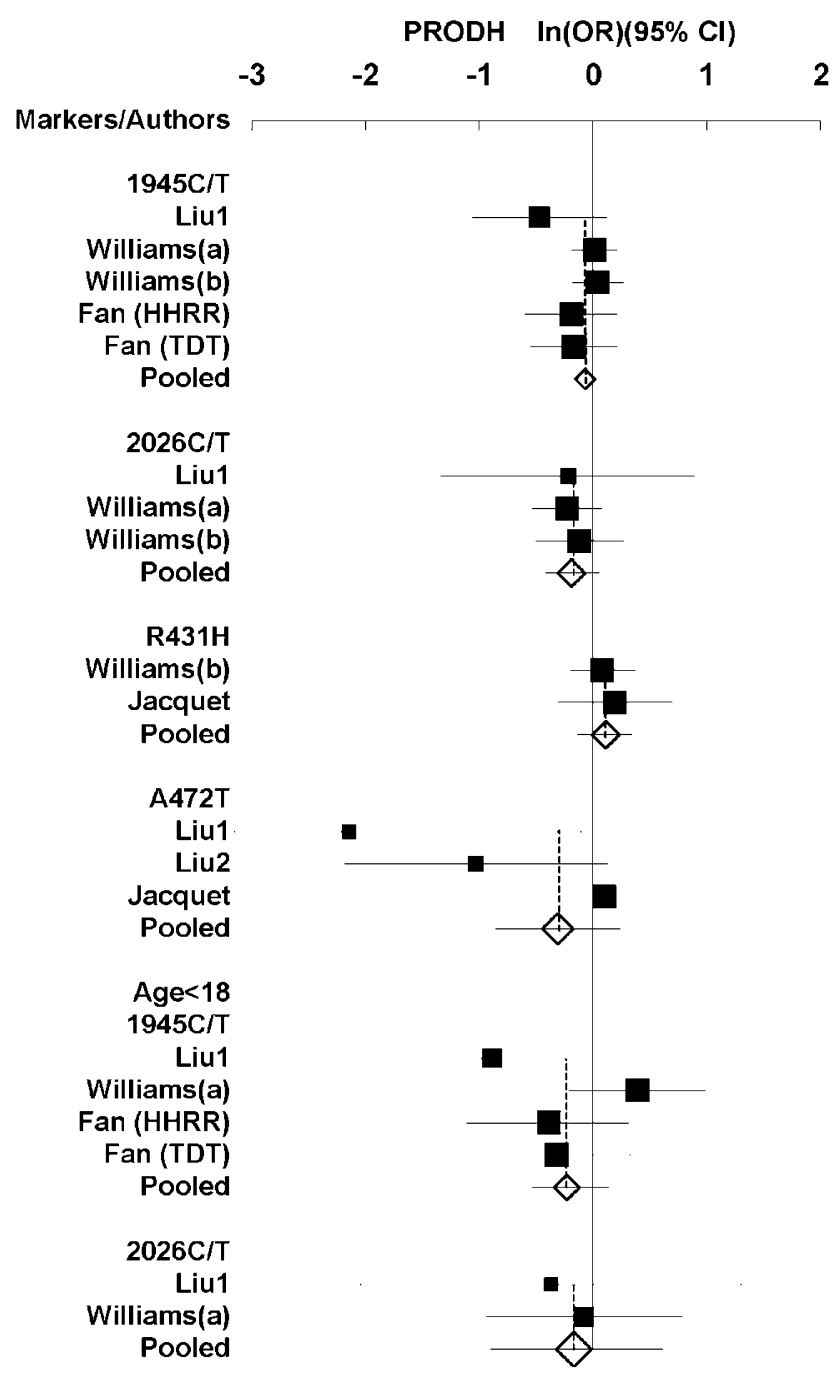

Figure 2 Forest plots of $\ln (\mathrm{OR})$ with $95 \% \mathrm{Cl}$ for each polymorphism of the allelic analysis for the $P R O D H$ gene.

or not significant when the data of the initial report ${ }^{15}$ were excluded (Table 1). The results suggested that the original finding might be false and that RGS4 is not a susceptibility gene. Thirdly, both genes were expressed in various tissues and brain regions and therefore were likely to be under complex expression regulations affected by different SNP combinations. Fourthly, genetic structure or environmental factors such as the season of birth, which may be associated with several psychiatric and neurological disorders, ${ }^{37}$ may also result in variability. Actually, such variability is not unique to RGS4 and $P R O D H$, as other genes, like the DTNBP1 gene (unpublished), have also shown different allelic associations with schizophrenia.

As for the LD and haplotype structure (Utah residents), the four SNPs of $R G S 4$ were in the $5^{\prime}$ end of the 

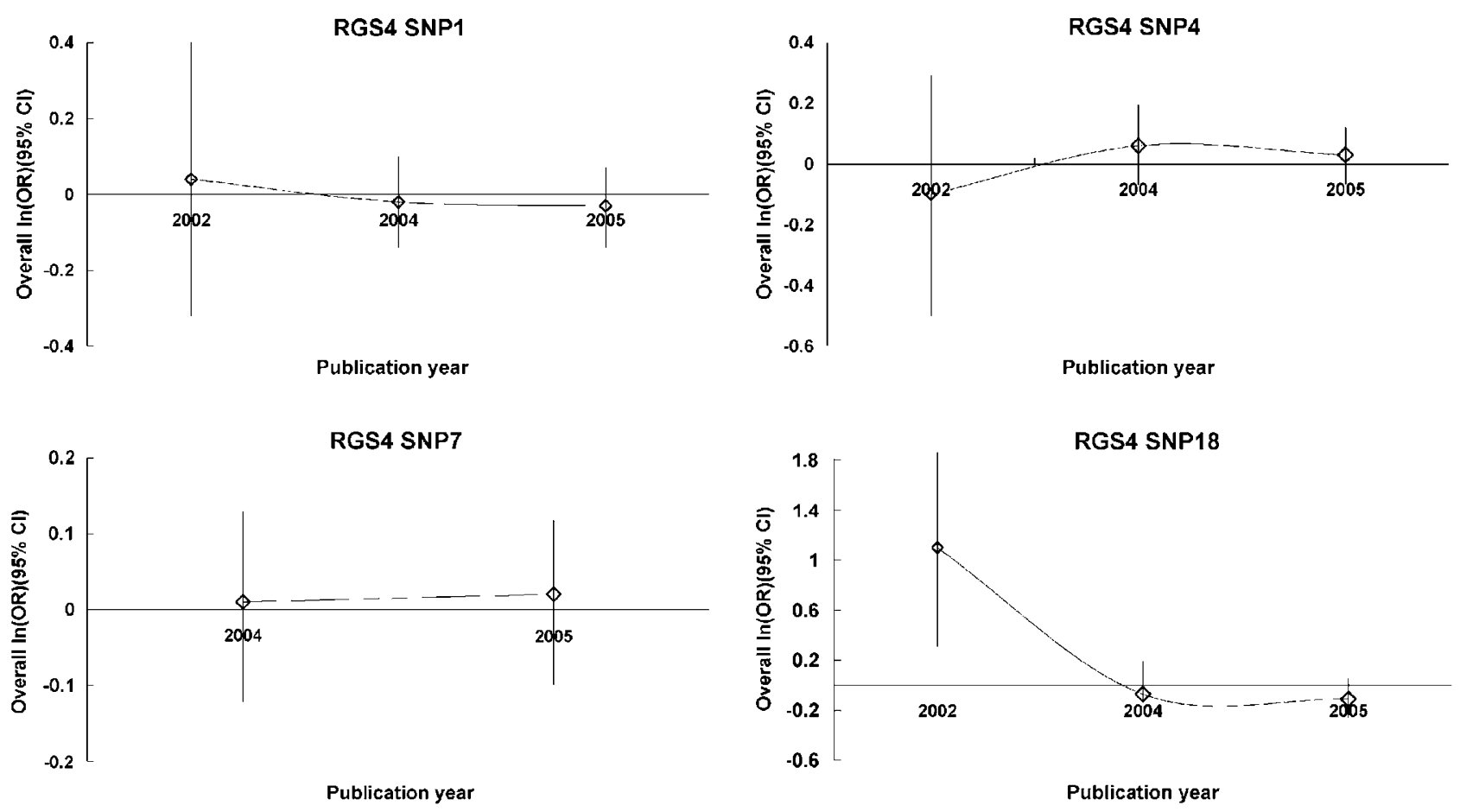

Figure 3 Retrospective analysis. Analysis in retrospect, based on publication year since 2002, is shown for RGS4, which was similar to that of $P R O D H$ (not shown).

gene whereas those of $P R O D H$ were in the $3^{\prime}$ direction (Supplementary figures). For each gene, the four SNPs were in a small strong LD structure. The whole RGS4 gene was in a large LD structure, which was also supported by previous studies. ${ }^{30,31}$ Although all the polymorphisms tested were negative, this may not be sufficient to rule out the possibility of association of other risk polymorphisms, which locate out of the strong LD structure for each gene, considering the positive evidence in previous studies. Further investigation of other at-risk polymorphisms or haplotypes, particularly exhaustive analyses at each locus, is necessary. Another likely possibility is that they are small effect genes. For subsequent association studies, accurate phenotype definition, strict selection of patients, much larger samples and accurate phenotypic data will be required, to facilitate comparability between study outcomes, and the pooling of data in future meta-analyses.

This may be the first meta-analysis focusing on RGS4 and $P R O D H$. However, it has some limitations such as the small sample size for $\mathrm{R} 431 \mathrm{H}$, and the fact that a haplotypic meta-analysis could not be conducted as haplotype data were available in only two studies for each gene. Schizophrenia is highly heritable, ${ }^{38}$ and it may result from the combined effects of multiple susceptibility loci. However, the nature of schizophrenia remains largely unknown, and the task of locating and identifying relevant major genes remains problematical.

\section{Acknowledgements}

This work was supported by grants from the Ministry of Education, PRC, the national 973 and 863 programs, the National Natural Science Foundation of China and the Shanghai Municipal Commission for Science and Technology.

\section{Electronic-database information}

Accession Numbers and URLs for data in this article are as follows: Online Mendelian Inheritance in Man (OMIM), http://www.ncbi.nlm. nih.gov/Omim for RGS4 and PRODH.

Genotype data, http://www.hapmap.org/ for RGS4 and PRODH.

Genome data, http://genome.ucsc.edu/ for RGS4 and PRODH.

\section{References}

1 Cannon TD, Kaprio J, Lonnqvist J, Huttunen M, Koskenvuo M: The genetic epidemiology of schizophrenia in a Finnish twin cohort. A population-based modeling study. Arch Gen Psychiatry 1998; 55: 67-74.

2 Jablensky A: Epidemiology of schizophrenia: the global burden of disease and disability. Eur Arch Psychiatry Clin Neurosci 2000; 250: $274-285$.

3 Rapoport JL, Addington AM, Frangou S, Psych MR: The neurodevelopmental model of schizophrenia: update 2005. Mol Psychiatry 2005; 10: 434-449.

4 Collier DA, Li T: The genetics of schizophrenia: glutamate not dopamine? Eur J Pharmacol 2003; 480: 177-184.

5 Harrison PJ, Owen MJ: Genes for schizophrenia? Recent findings and their pathophysiological implications. Lancet 2003; 361: 417-419.

6 Karayiorgou M, Gogos JA: The molecular genetics of the 22q11-associated schizophrenia. Brain Res Mol Brain Res 2004; 132: $95-104$. 
7 Owen MJ, Williams NM, O'Donovan MC: The molecular genetics of schizophrenia: new findings promise new insights. Mol Psychiatry 2004; 9: 14-27.

8 McGuffin P, Tandon K, Corsico A: Linkage and association studies of schizophrenia. Curr Psychiatry Rep 2003; 5: 121-127.

9 Brzustowicz LM, Hodgkinson KA, Chow EW, Honer WG, Bassett AS: Location of a major susceptibility locus for familial schizophrenia on chromosome 1q21-q22. Science 2000; 288: 678-682.

10 De Blasi A, Conn PJ, Pin J, Nicoletti F: Molecular determinants of metabotropic glutamate receptor signaling. Trends Pharmacol Sci 2001; 22: 114-120.

11 Mirnics K, Middleton FA, Stanwood GD, Lewis DA, Levitt P: Disease-specific changes in regulator of G-protein signaling 4 (RGS4) expression in schizophrenia. Mol Psychiatry 2001; 6: 293-301.

12 Thaminy S, Auerbach D, Arnoldo A, Stagljar I: Identification of novel ErbB3-interacting factors using the split-ubiquitin membrane yeast two-hybrid system. Genome Res 2003; 13: 1744-1753.

13 Tkachev D, Mimmack ML, Ryan MM et al: Oligodendrocyte dysfunction in schizophrenia and bipolar disorder. Lancet 2003; 362: 798-805.

14 Stefansson H, Sigurdsson E, Steinthorsdottir V et al: Neuregulin 1 and susceptibility to schizophrenia. Am J Hum Genet 2002; 71: $877-892$.

15 Chowdari KV, Mirnics K, Semwal P et al: Association and linkage analyses of RGS4 polymorphisms in schizophrenia. Hum Mol Genet 2002; 11: 1373-1380.

16 Murphy KC, Jones LA, Owen MJ: High rates of schizophrenia in adults with velo-cardio-facial syndrome. Arch Gen Psychiatry 1999; 56: 940-945.

17 Jacquet H, Raux G, Thibaut F et al: PRODH mutations and hyperprolinemia in a subset of schizophrenic patients. Hum Mol Genet 2002; 11: 2243-2249.

18 Williams HJ, Williams N, Spurlock G et al: Detailed analysis of PRODH and PsPRODH reveals no association with schizophrenia. Am J Med Genet B Neuropsychiatr Genet 2003; 120: 42-46.

19 O'Donovan MC, Williams NM, Owen MJ: Recent advances in the genetics of schizophrenia. Hum Mol Genet 2003; 12 (Spec. No. 2): R125-R133.

20 Gogos JA, Santha M, Takacs Z et al: The gene encoding proline dehydrogenase modulates sensorimotor gating in mice. Nat Genet 1999; 21: 434-439.

21 Pearlson GD: Neurobiology of schizophrenia. Ann Neurol 2000; 48: 556-566.

22 Liu H, Heath SC, Sobin C et al: Genetic variation at the 22q11 PRODH2/DGCR6 locus presents an unusual pattern and increases susceptibility to schizophrenia. Proc Natl Acad Sci USA 2002; 99: $3717-3722$

23 Cho HJ, Meira-Lima I, Cordeiro Q et al: Population-based and family-based studies on the serotonin transporter gene polymorphisms and bipolar disorder: a systematic review and metaanalysis. Mol Psychiatry 2005; 10: 771-781.
24 Egger M, Davey Smith G, Schneider M, Minder C: Bias in meta-analysis detected by a simple, graphical test. BMJ 1997; 315 : $629-634$.

25 Chen X, Dunham C, Kendler S et al: Regulator of G-protein signaling 4 (RGS4) gene is associated with schizophrenia in Irish high density families. Am J Med Genet B Neuropsychiatr Genet 2004; 129: $23-26$.

26 Ohtsuki T, Tanaka S, Ishiguro H et al: Failure to find association between PRODH deletion and schizophrenia. Schizophr Res 2004; 67: $111-113$.

$27 \mathrm{Li}$ T, Ma X, Sham PC et al: Evidence for association between novel polymorphisms in the PRODH gene and schizophrenia in a Chinese population. Am J Med Genet B Neuropsychiatr Genet 2004; 129: $13-15$

28 Bender HU, Almashanu S, Steel G et al: Functional consequences of PRODH missense mutations. Am J Hum Genet 2005; 76: 409-420.

29 Hoogendoorn B, Coleman SL, Guy CA, Smith SK, O'Donovan MC, Buckland PR: Functional analysis of polymorphisms in the promoter regions of genes on 22q11. Hum Mutat 2004; 24: $35-42$.

30 Morris DW, Rodgers A, McGhee KA et al: Confirming RGS4 as a susceptibility gene for schizophrenia. Am J Med Genet $B$ Neuropsychiatr Genet 2004; 125: 50-53.

31 Williams NM, Preece A, Spurlock G et al: Support for RGS4 as a susceptibility gene for schizophrenia. Biol Psychiatry 2004; 55: 192-195.

32 Cordeiro Q, Talkowski ME, Chowdari KV, Wood J, Nimgaonkar V, Vallada H: Association and linkage analysis of RGS4 polymorphisms with schizophrenia and bipolar disorder in Brazil. Genes Brain Behav 2005; 4: 45-50.

33 Williams HJ, Williams N, Spurlock G et al: Association between PRODH and schizophrenia is not confirmed. Mol Psychiatry 2003; 8: $644-645$.

34 Fan JB, Ma J, Zhang CS et al: A family-based association study of T1945C polymorphism in the proline dehydrogenase gene and schizophrenia in the Chinese population. Neurosci Lett 2003; 338: 252-254.

35 Jacquet H, Demily C, Houy E et al: Hyperprolinemia is a risk factor for schizoaffective disorder. Mol Psychiatry 2005; 10: $479-485$.

36 Thaler RH: The Winner's Curse Paradoxes and Anomalies of Economic Life. Princeton, NJ: Princeton University Press, 1999.

37 Chotai J, Serretti A, Lattuada E, Lorenzi C, Lilli R: Geneenvironment interaction in psychiatric disorders as indicated by season of birth variations in tryptophan hydroxylase (TPH), serotonin transporter (5-HTTLPR) and dopamine receptor (DRD4) gene polymorphisms. Psychiatry Res 2003; 119: 99-111.

38 Styner M, Lieberman JA, McClure RK, Weinberger DR, Jones DW, Gerig G: Morphometric analysis of lateral ventricles in schizophrenia and healthy controls regarding genetic and diseasespecific factors. Proc Natl Acad Sci USA 2005; 102: 4872-4877.

Supplementary Information accompanies the paper on European Journal of Human Genetics website (http://www.nature.com/ejhg) 